\title{
Accuracy of ultrasonography and color Doppler in diagnosis of ovarian masses and its correlation with histopathological findings
}

\author{
Kariman Ghazal $^{1,2 *}$, Jihad El Hasan ${ }^{1,3}$, Hanine Hijasi² ${ }^{2}$ Ev Koulaima ${ }^{3}$
}

\author{
${ }^{1}$ Department of Obstetrics and Gynecology, Lebanese University, Lebanon \\ ${ }^{2}$ Department of Obstetrics and Gynecology, Rafik al Hariri Hospital University Medical Center, Beirut, Lebanon \\ ${ }^{3}$ Department of Obstetrics and Gynecology, Al Zahraa Hospital University Medical Center, Beirut, Lebanon
}

Received: 23 September 2020

Revised: 31 October 2020

Accepted: 02 November 2020

\section{*Correspondence: \\ Dr. Kariman Ghazal, \\ E-mail: ghazal_kariman@hotmail.it}

Copyright: (C) the author(s), publisher and licensee Medip Academy. This is an open-access article distributed under the terms of the Creative Commons Attribution Non-Commercial License, which permits unrestricted non-commercial use, distribution, and reproduction in any medium, provided the original work is properly cited.

\section{ABSTRACT}

Background: Ovarian cancer is often diagnosed in advanced stages due to the absence of specific symptoms. Yet, there are no reliable screening tests for ovarian malignancies. However, improvements in imaging techniques such as ultrasonography and color Doppler increased the probability of early detection. The purpose of this study was to compare between the ultrasonographic and color Doppler findings of ovarian masses with their histopathological diagnosis.

Methods: A retrospective review was performed on patients between January 2017 and January 2020 diagnosed to have an ovarian mass. The eligible patients were subsequently divided into 2 groups: group A included patients who had abdominal and vaginal ultrasound in order to assess the morphology of the ovarian neoformation, and group B included patients whose ovarian masses were evaluated by color Doppler in addition to transabdominal ultrasound in order to identify their vascularization as well as their morphology.

Results: A total of 74 patients were included in the study with 42 patients in group A and 32 patients in group B. Of the 42 adnexal masses studied in group A, 8 (19.0\%) were malignant, $33(78.6 \%)$ were benign and $1(2.4 \%)$ was borderline. Ultrasound had a sensitivity of $89.0 \%$, a specificity of $100.0 \%$, a positive predictive value (PPV) of $100.0 \%$, a negative predictive value (NPV) of $97.0 \%$ and accuracy of $97.0 \%$. On the other hand, of the 32 masses examined in Group B, 7 (21.9\%) were malignant, $23(71.9 \%)$ were benign and $2(6.3 \%)$ were borderline. Color doppler had a sensitivity of $55.6 \%$, a specificity of $95.5 \%$, a PPV of $83.0 \%$, a NPV of $84.0 \%$ and accuracy of $84.0 \%$. When using color Doppler in addition to morphology score, the sensitivity was $100.0 \%$, specificity $95.5 \%$, PPV $90.0 \%$, NPV $100.0 \%$ and accuracy $96.6 \%$.

Conclusions: Ultrasound combined to color Doppler improves the diagnosis of ovarian tumors with accurately detecting malignant from benign ones.

Keywords: Ovarian cancer, Ultrasonography, Doppler

\section{INTRODUCTION}

Ovarian cancer is the eighth most common cancer in women with an incidence of 295414 cases and a rate of 7.8 per 100,000 women identified in 2018 , accounting for $3.4 \%$ of female malignant tumors. ${ }^{1}$ It contributes to women mortality with a rate of 4.9 per $100,000 .{ }^{1}$ This relatively high mortality rate is associated with delayed onset of symptoms and lack of appropriate screening tools which lead to its diagnosis in advanced stages. ${ }^{2}$ There are different histological types of ovarian cancer. The epithelial type being the prevailing one. ${ }^{3}$ Various risk factors affect the development of ovarian cancer. Age and 
genetic predisposition are among the most important factors. $^{4}$

Despite the progress made in the implementation of accurate staging and therapeutic strategies, the main prognostic factor remains the stage of the disease at the time of diagnosis. ${ }^{2}$ There is a crucial need to make an early diagnosis to obtain a real benefit in terms of survival. ${ }^{5}$ Several early detection methods for ovarian cancer are being used; however, the most effective screening technique remains controversial. ${ }^{6}$ The first studies with transabdominal pelvic ultrasound have allowed to standardize the morphological ultrasound criteria to make a differential diagnosis between benign and malignant cystic ovarian mass. ${ }^{7}$ Persisting difficulty in making early diagnosis of malignant tumor led to the use of both morphology and ovarian volume depending on menopausal status. ${ }^{7}$ Then, the transvaginal pathway was utilized in screening since it is more sensitive and specific than transabdominal ultrasound. Transabdominal ultrasound should however not be considered outdated as it offers a complete overview of the pelvic anatomy. ${ }^{8}$ The transvaginal ultrasound, on the other hand, allows to delineate the fine structural details of pelvic masses and to identify any abnormal finding within myometrium or endometrium that might be possibly associated with adnexal abnormality. ${ }^{8}$

Following that, Doppler flow measurement was introduced in order to improve the specificity and sensitivity of the ultrasound. This technique is based on the consideration that all neoplasms, including ovarian ones, present a modification of the vascularization resulting from angiogenesis phenomenon. ${ }^{9}$

The color doppler allows to examine the flow of the pelvic organs investigated by ultrasound. ${ }^{10-12}$ Combining the advantages of color with high resolution image provides a proper and immediate visualization of the pelvic vessels. With the aid of the color doppler all the vessels are identified. Therefore, it is possible to distinguish the vessels characterized by high resistance and low flow, typical of a benign lesion, and low resistance vessels with high flow, a characteristic of malignant pathological process. $^{11}$

Consequently, the effectiveness of color doppler in addition to conventional ultrasonography has shown promising outcomes in differentiating ovarian masses and diagnosing malignancy at an early stage. ${ }^{11}$ Hence, the purpose of this study was to assess the ultrasonographic and color doppler findings of both malignant and benign ovarian masses and compare them with their histopathological diagnosis.

\section{METHODS}

A retrospective review was performed on patients who visited the obstetrics and gynecology clinic between January 2017 and January 2020 diagnosed with an ovarian mass. Inclusion criteria were: women of all ages including pre-pubertal and post-menopaused patients having palpable mass and symptoms such as pain in abdomen/pelvis, bleeding, urinary and gastrointestinal pressure, as well as asymptomatic patients whose pelvic masses were detected at the time of a routine pelvic examination or incidental findings at the time of transabdominal and transvaginal sonography done for nongynecological purposes. Exclusion criteria were patients on ovulation induction drugs, who did not undergo fine needle aspiration cytology or histopathological evaluation, and patients with ectopic or normal pregnancy.

The eligible patients were subsequently divided into two groups: group A included patients who underwent abdominal and vaginal ultrasound for the purpose of studying the morphology of the pelvic neoformation. On the other hand, group B included patients whose ovarian masses were evaluated by color Doppler in addition to transabdominal ultrasound in order to identify their vascularization as well as their morphology.

A Samsung real time ultrasound system using a 3.5 Mhz convex probe was used to carry out the examination via the transabdominal route for all patients with full bladder. An 8-10 Mhz probe was used to carry out the tests by transvaginal route with empty bladder. Virgin patients had transabdominal ultrasound exclusively. A morphological examination and assessment of the ovarian masses was done for both groups with particular regard to the following characteristics: size, content, walls, septa, vegetation and peritoneal liquid. A morphology index which is based on morphologic characteristics including tumor volume, wall structure, and septal structure, was used in order to differentiate between benign and malignant masses. A score less than 9 labels the mass as benign. $^{13}$

In group $\mathrm{B}$, in addition to morphology, the presence of vascular flows was assessed by transabdominal color Doppler. For each mass, the site of the vascularization was indicated: central (within solid areas, tokens, papillary or crossing of the septa) or peripheral (at the level of the capsule, in the cystic lesions, or at the peripheral level in the solid masses). The pulsatility index (PI), the resistance index (RI), and the peak systolic velocity (VPS) were recorded by registering the lowest value of $\mathrm{Pl}$ and $\mathrm{RI}$ and the maximum peak speed which differentiate between benign and malignant tumors. RI $<0.4$ and PI $<1.0$ were considered as cutoff for ovarian malignancy. ${ }^{14}$ The results of the ultrasound, morphological and Doppler investigations were subsequently compared with the surgical, laparotomy, or histopathological findings.

Figure 1 shows transvaginal ultrasound of a 39-year-old woman G2P2 complaining of lower abdominal pain. She used contraceptive for many years. She was followed for several months with the cyst persisting. Morphological score $<10 \mathrm{~cm}$, Doppler negative, Ca 125=39. She underwent laparotomy cystectomy. This mass measured 
$64 \mathrm{~mm}$ by $42 \mathrm{~mm}$. The smooth cyst contained clear citrine fluid. Pathology revealed a benign cystadenoma (simple cyst).
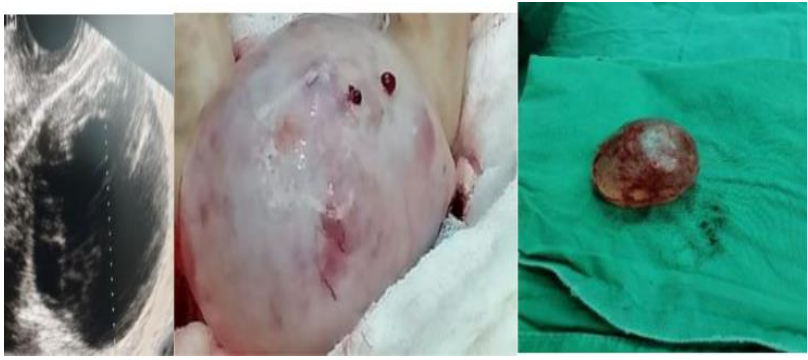

Figure 1: Transvaginal ultrasound of a 39-year-old woman.

Figure 2 shows transabdominal ultrasound of a 64-yearold woman with pelvic mass. It measured $>10 \mathrm{~cm}$ and contained septations and papillary projections. Morphological score $>10$, Ca 125>234. No Doppler was done. Surgery revealed a first stage adenocarcinoma of the ovary. Histology confirmed an adenocarcinoma.

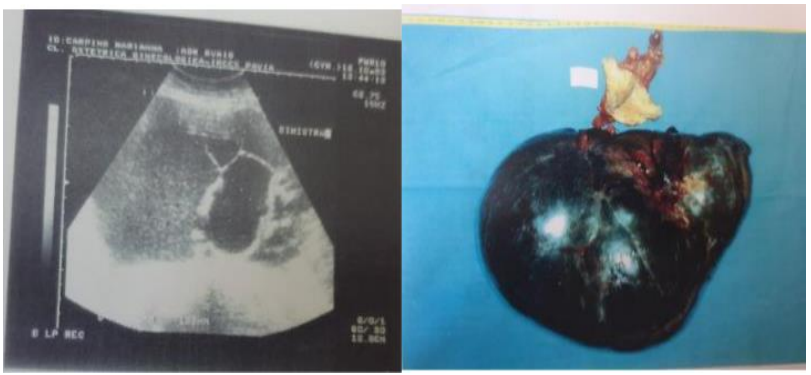

Figure 2: Transabdominal ultrasound of a 64-year-old woman with pelvic mass.

Figure 3 shows transvaginal ultrasound of a 67-year-old woman with pelvic mass measuring $>7 \mathrm{~cm}$ and containing solid content, $\mathrm{Ca} 125=60$. A vascular flow was noted inside a solid part. Clear citrine fluid was seen during operation. Surgery revealed a cystadenoma of the ovary confirmed by histology.

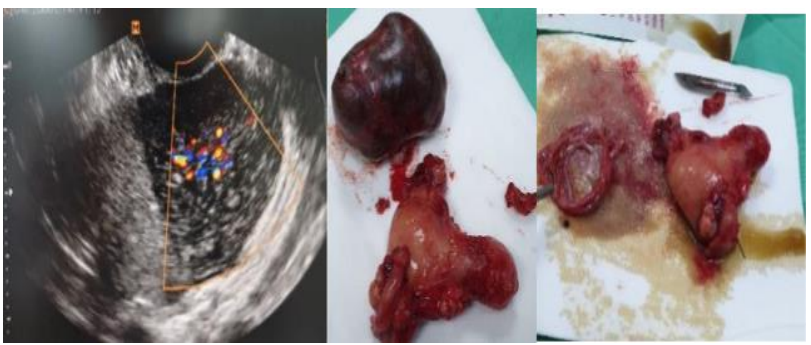

Figure 3: Transvaginal ultrasound of a 67-year-old woman with pelvic mass.

Figure 4 shows abdominal ultrasound of a 61-year-old woman with pelvic mass measuring $>10 \mathrm{~cm}$ and containing septations and solid components. Papillary projections were also noted. Morphological score $>10$, Ca 125>234. Doppler revealed vascular flow inside the mass. Surgery revealed a stage 2 adenocarcinoma of the ovary confirmed by histology.
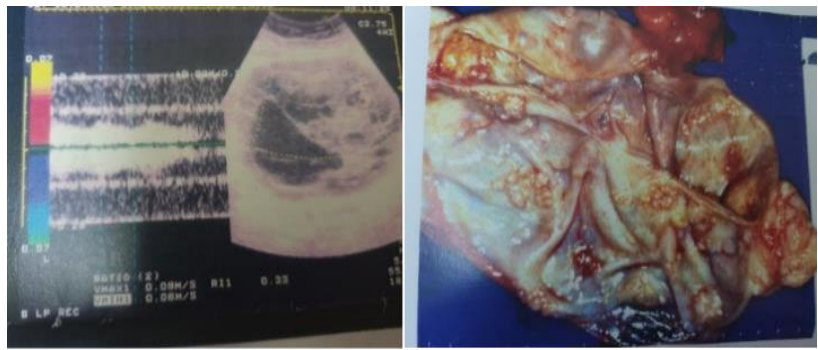

Figure 4: Abdominal ultrasound of a 61-year-old woman with pelvic mass.

Figure 5 shows transvaginal ultrasound of a 64-year-old woman with pelvic mass measuring $>10 \mathrm{~cm}$, containing septation and papillary projections. Ca 125>234. Doppler showed a vascular flow inside the mass. Surgery revealed a stage 1 adenocarcinoma of the ovary confirmed by histology.


Figure 5: Transvaginal ultrasound of a 64-year-old woman with pelvic mass.

Figure 6 shows transabdominal ultrasound of a 17-yearold girl complaining of severe pain of 1-month duration and amenorrhea. The cyst measured $20 \mathrm{~mm}$, contained mostly echoic structure and produced a noticeable acoustic shadow with attenuation of the sound. Morphological score $>10$. Doppler showed no vascular flow inside the mass. Laparotomy was done to show a dermoid cyst of the ovary with a subsequent ovarian torsion confirmed by histology.
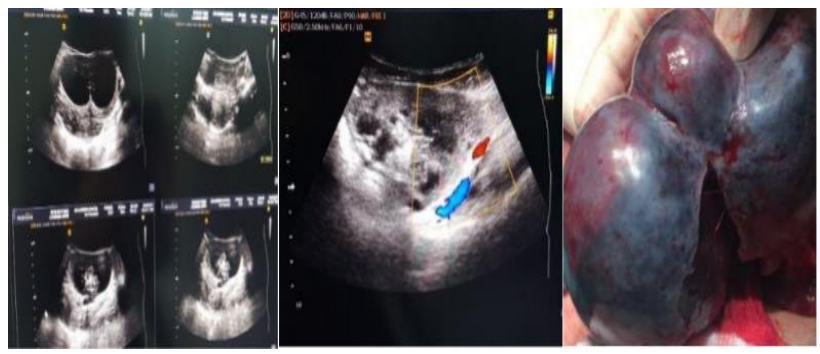

Figure 6: Transabdominal ultrasound of a 17-year-old girl. 
Figure 7 shows transvaginal ultrasound of a 63-year-old woman with pelvic mass measuring $>10 \mathrm{~cm}$ and containing septations and papillary projections. Doppler showed a vascular flow in the mass wall with little flow inside. $\mathrm{Ca}$ $125>23$. Surgery revealed an adenocarcinoma of the ovary. Histology confirmed stage 1 malignancy.

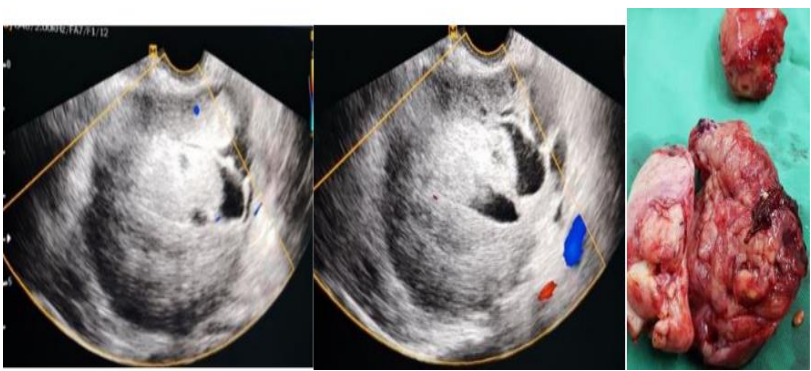

Figure 7: Transvaginal ultrasound of a 63-year-old woman with pelvic mass.

Figure 8 shows ovarian cyst observed on transabdominal ultrasound in a 25-year-old woman measuring $70 \mathrm{~mm}$ who presented with pelvic pain. Typical appearance of dermoid was noticed. Doppler showed no flow within the cyst. Laparotomy was done and pathology confirmed the diagnosis of dermoid cyst associated with another hemorrhagic cyst in addition to a para-ovarian cyst.

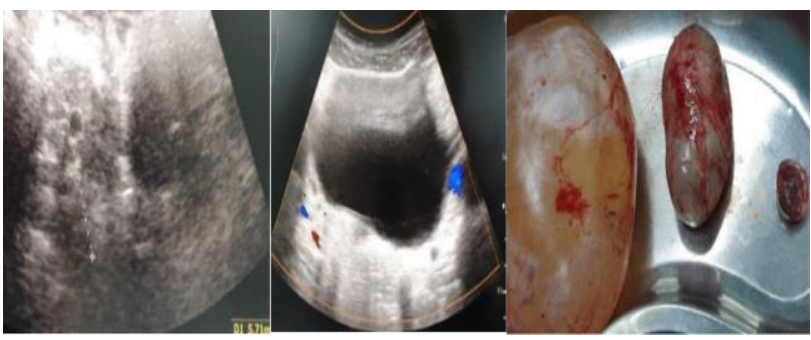

Figure 8: Ovarian cyst observed on transabdominal ultrasound in a 25-year-old woman.

Figure 9 shows transabdominal ultrasound of a 27-yearold woman with pelvic mass measuring $>30 \mathrm{~cm}$ and containing solid components with septations and papillary projections, Ca $125=60$. Vascular flow was noted on part of the wall without any flow inside the mass. Surgery was done and pathology revealed immature teratoma of the ovary with intestinal components containing calcifications and foci of fat consistency.
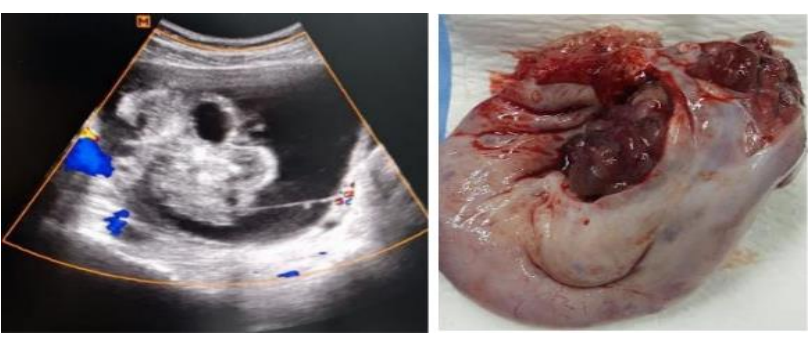

Figure 9: Transabdominal ultrasound of a 27-year-old woman with pelvic mass.
Figure 10 shows transvaginal ultrasound scan of a 40-yearold woman multiparous with irregular menstrual cycle and severe pain not resolving for 1 year. A heterogeneous mass was noted. Doppler showed vascular flow, Ca 125>35. A hypoechogenic cyst with an echogenic structure representing the blood clot like "cobweb" was seen. Surgery revealed hemorrhagic cyst confirmed with histology.

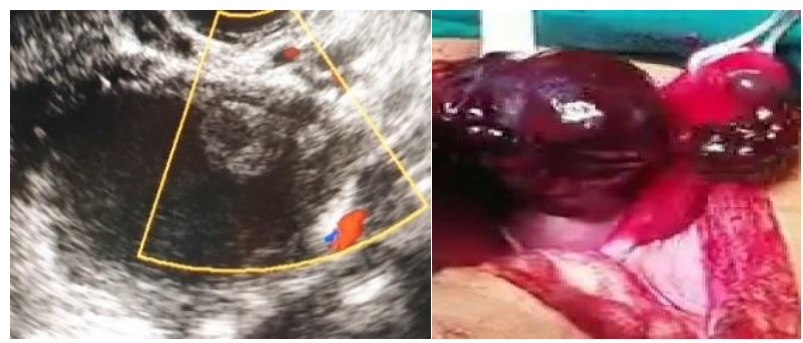

Figure 10: Transvaginal ultrasound scan of a 40-yearold woman.

Figure 11 shows transvaginal ultrasound scan of a 29-yearold woman multiparous with irregular menstrual cycle and severe pain revealed this image: thin walled cyst with fine internal reticular pattern without vascular flow inside suggestive of a hemorrhagic cyst. It was resolved with medical treatment and analgesics.

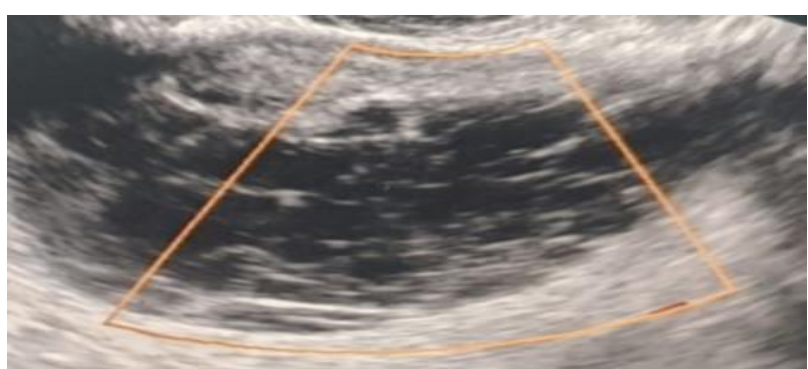

Figure 11: Transvaginal ultrasound scan of a 29-yearold woman.

Figure 12 shows transabdominal and transvaginal ultrasound scan of 25-year-old woman multiparous with irregular menstrual cycle presenting with severe pain. This image revealed No vascular flow within the cyst. Findings were suggestive with hemorrhagic cyst which was resolved with medical treatment.

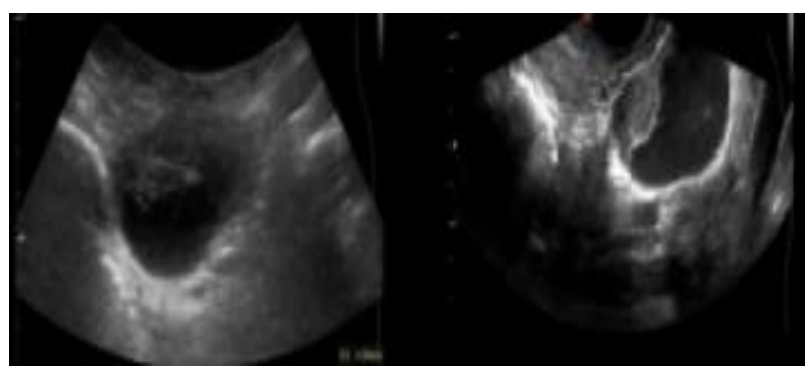

Figure 12: Transabdominal and transvaginal ultrasound scan of 25-year-old woman. 
Figure 13 shows transabdominal scan of 20-year-old woman multiparous with irregular menstrual cycle presenting with severe pain. This image revealed a simple cyst with no vascularity flow suggestive of luteinic cyst. It disappeared after menstrual cycle.

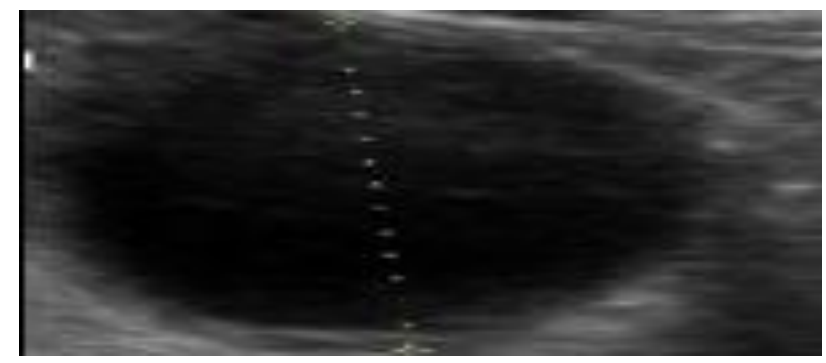

Figure 13: Transabdominal scan of 20-year-old woman.

Figure 14 shows transabdominal ultrasound scan of 15year-old woman with irregular cycle and severe pain. US images showed a right ovarian cyst with internal echoes and without a vascular flow within it. This was suggestive of hemorrhagic cyst. It was resolved with medical treatment and analgesics.

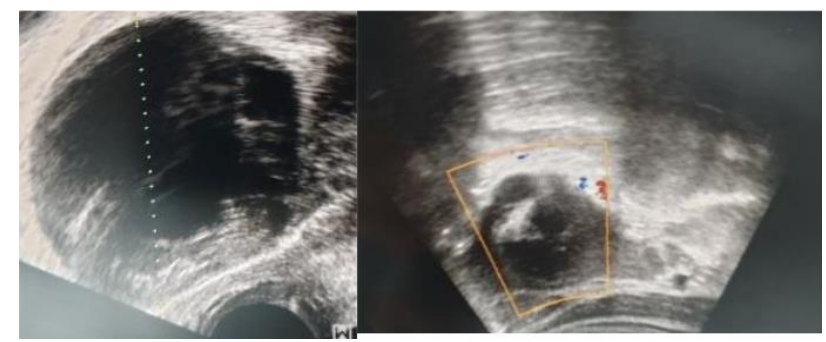

Figure 14: Transabdominal ultrasound scan of 15year-old woman.

Statistical analysis was performed using the statistical package for social sciences (SPSS version 24). Chi-square or Fisher's exact tests and independent sample t-test were used to compare categorical and continuous variables, respectively. Data were presented as a mean \pm standard deviation or number (percent) as appropriate. $\mathrm{P}$ value $<0.05$ was considered to indicate statistical significance.

\section{RESULTS}

A total of 74 patients were included in the study with 42 patients in group A and 32 patients in group B. There were no significant differences between the two groups in terms of mean age and parity (Table 1). Of the 42 adnexal masses studied in group A, 8 (19.0\%) were malignant, 33 (78.6\%) were benign and $1(2.4 \%)$ was borderline. The most common malignant mass was first stage adenocarcinoma $(62.5 \%)$ whereas the most common benign masses were serosal cysts $(30.3 \%)$ followed by ovarian endometriosis cyst and cystadenoma (simple) cysts (21.2\% each). On the other hand, of the 32 masses examined in group B, 7 $(21.9 \%)$ were malignant, $23(71.9 \%)$ were benign and 2 $(6.3 \%)$ were borderline. First stage adenocarcinoma was the highest among malignant neoplasms (57.1\%) while serosal cysts were the highest among benign tumors (39.1\%) followed by para-ovarian cysts $(17.4 \%$ ) (Table 1$)$.

The mean morphology scores for group A are shown in (Table 2). For the malignant cases, the highest mean scores

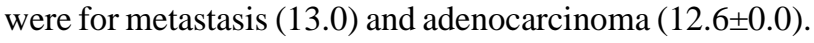
As for the benign cases, the mean score for thecoma and para-ovarian cyst was $6.0 \pm 0.0$ and for fibroma $4.6 \pm 0.0$.

Table 3 presents the characteristics of group B masses in terms of morphology score, as well as resistance index (RI), pulsatility index (PI) and peak systolic velocity (PSV) obtained by color Doppler. The site of vascularization was found to be mainly central in malignant lesions and predominantly peripheral in benign lesions. The absence of flow has been highlighted especially in benign lesions. The average value of the resistance index was $0.4 \pm 0.1$ for malignant lesions and $0.5 \pm 0.04$ for benign ones. The mean pulsatility index was $0.9 \pm 0.5$ for malignant tumors and $1.0 \pm 0.2$ for benign ones. The mean peak systolic velocity was $17.7 \pm 12.7 \mathrm{~cm} / \mathrm{s}$ for malignant masses and $6.4 \pm 0.9 \mathrm{~cm} / \mathrm{s}$ for benign masses.

From the analysis of the results obtained by evaluating only the ultrasound morphological aspect of the masses, it was possible to highlight that this method has a sensitivity of $89.0 \%$, a specificity of $100.0 \%$, a positive predictive value (PPV) of $100.0 \%$, a negative predictive value (NPV) of $97.0 \%$ and accuracy of $97.0 \%$ (Table 4). From the analysis of the results obtained by color doppler only, it was shown that this method has a sensitivity of $55.6 \%$, a specificity of $95.5 \%$, a PPV of $83.0 \%$, a NPV of $84.0 \%$ and accuracy of $84.0 \%$. When combining color doppler to ultrasound morphology score, the sensitivity was $100.0 \%$, specificity $95.5 \%$, PPV 90.0\%, NPV $100.0 \%$ and accuracy $96.6 \%$ (Table 4).

Table 1: Patients' demographic characteristics and type of masses.

\begin{tabular}{|lllll|}
\hline Characteristics & Overall & Group A (n=42) & Group B (n=32) & P value \\
\hline Age (years) & $37.7 \pm 15.2$ & $36.9 \pm 14.4$ & $38.8 \pm 16.3$ & 0.61 \\
\hline Parity & $2.1 \pm 1.4$ & $2.0 \pm 1.4$ & $2.3 \pm 1.3$ & 0.43 \\
\hline Malignant masses (\%) & $15(20.3)$ & $8(19.0)$ & $7(21.9)$ & 0.78 \\
\hline Type of malignancy (\%) & & & & 0.71 \\
\hline Metastasis & $3(20.0)$ & $1(12.5)$ & $2(28.6)$ & \\
\hline Sarcoma & $2(13.3)$ & $1(12.5)$ & $1(14.3)$ & \\
\hline
\end{tabular}




\begin{tabular}{|c|c|c|c|c|}
\hline Characteristics & Overall & Group A (n=42) & Group B (n=32) & P value \\
\hline Adenocarcinoma & $9(60.0)$ & $5 \quad(62.5)$ & $4(57.1)$ & \\
\hline Dysgerminoma & $1(6.7)$ & $1(12.5)$ & $0(0.0)$ & \\
\hline Benign masses (\%) & $56(75.7)$ & $33(78.6)$ & $23(71.9)$ & 0.70 \\
\hline Type of benign masses (\%) & & & & 0.54 \\
\hline Endometriosis cyst & $10(17.9)$ & 7 (21.2) & $3(13.0)$ & \\
\hline Hemorrhagic cyst & $2(3.6)$ & $2(6.1)$ & $0 \quad(0.0)$ & \\
\hline Cystadenoma & $9(16.1)$ & $7 \quad(21.2)$ & $2(8.7)$ & \\
\hline Serosal cyst & 19 (33.9) & $10(30.3)$ & $9(39.1)$ & \\
\hline Dermoid cyst & $3(5.4)$ & $1(3.0)$ & $2(8.7)$ & \\
\hline Thecoma & $2(3.6)$ & $1(3.0)$ & $1(4.3)$ & \\
\hline Fibroma & $5(8.9)$ & $3(9.1)$ & $2(8.7)$ & \\
\hline Para-ovarian cyst & $6(10.7)$ & $2(6.1)$ & $4(17.4)$ & \\
\hline Borderline masses (\%) & $3(4.1)$ & $1 \quad(2.4)$ & $2(6.3)$ & 0.58 \\
\hline
\end{tabular}

Data are presented as number (percent) or mean \pm standard deviation

Table 2: Characteristics of group A masses.

\begin{tabular}{|lll|}
\hline Type & Number & Morphology score $($ mean \pm SD) \\
\hline Malignant & & 9.0 \\
\hline Borderline & 1 & 13.0 \\
\hline Metastasis & 1 & 11.0 \\
\hline Sarcoma & 1 & $12.6 \pm 0.0$ \\
\hline Adenocarcinoma & 5 & 11.0 \\
\hline Dysgerminoma & 1 & \\
\hline Benign & & $4.0 \pm 0.0$ \\
\hline Endometriosis cyst & 7 & $2.5 \pm 0.0$ \\
\hline Hemorrhagic cyst & 2 & $4.4 \pm 0.0$ \\
\hline Cystadenoma & 7 & $2.4 \pm 0.0$ \\
\hline Serosal cyst & 10 & 8.0 \\
\hline Dermoid cyst & 1 & 6.0 \\
\hline Thecoma & 1 & $4.6 \pm 0.0$ \\
\hline Fibroma & 3 & $6.0 \pm 0.0$ \\
\hline Para-ovarian cyst & 2 &
\end{tabular}

Table 3: Characteristics of group B masses.

\begin{tabular}{|llllll|}
\hline Type & Number & $\begin{array}{l}\text { Morphology } \\
(\mathbf{m e a n} \pm \mathbf{S D})\end{array}$ & $\begin{array}{l}\text { RI } \\
(\mathbf{m e a n} \pm \mathbf{S D})\end{array}$ & $\begin{array}{l}\text { PI } \\
(\mathbf{m} \text { ean } \pm \text { SD })\end{array}$ & $\begin{array}{l}\text { PSV } \\
(\mathbf{m e a n} \pm \mathbf{S D})\end{array}$ \\
\hline Malignant & & & $0.4 \pm 0.1$ & $0.9 \pm 0.5$ & $17.7 \pm 12.7$ \\
\hline Metastasis & 2 & $11.5 \pm 0.7$ & $0.4 \pm 0.0$ & - & $25.0 \pm 0.0$ \\
\hline Fibroleiomyosarcoma & 1 & 13.0 & 0.5 & 1.2 & 3.0 \\
\hline Adenocarcinoma & 4 & $13.3 \pm 1.2$ & $0.4 \pm 0.0$ & $0.5 \pm 0.0$ & $25.0 \pm 0.0$ \\
\hline Borderline & 2 & $11.0 \pm 0.3$ & $0.3 \pm 0.0$ & $0.5 \pm 0.0$ & $10.0 \pm 0.3$ \\
\hline Benign & & & $0.5 \pm 0.04$ & $1.0 \pm 0.2$ & $6.4 \pm 0.9$ \\
\hline Endometriosis cyst & 3 & $4.0 \pm 0.0$ & $0.4 \pm 0.1$ & $1.0 \pm 0.2$ & $7.3 \pm 2.3$ \\
\hline Cystadenoma & 2 & $4.4 \pm 0.0$ & $0.5 \pm 0.0$ & $1.0 \pm 0.0$ & $6.0 \pm 0.0$ \\
\hline Serosal cyst & 9 & $2.6 \pm 0.7$ & $0.6 \pm 0.1$ & $1.3 \pm 0.2$ & $7.1 \pm 1.1$ \\
\hline Dermoid cyst & 2 & $7.0 \pm 1.4$ & $0.5 \pm 0.0$ & $1.0 \pm 0.0$ & $7.0 \pm 0.0$ \\
\hline Thecoma & 1 & 6.6 & 0.5 & 1.0 & 7.0 \\
\hline Fibroma & 2 & $8.3 \pm 3.9$ & $0.5 \pm 0.0$ & $0.7 \pm 0.4$ & $5.5 \pm 6.4$ \\
\hline Para-ovarian cyst & 4 & $3.8 \pm 0.3$ & $0.5 \pm 0.0$ & $1.0 \pm 0.0$ & $5.0 \pm 0.0$ \\
\hline
\end{tabular}

*RI: resistance index, PI: pulsatility index, PSV: peak systolic velocity. 
Table 4: Sensitivity and specificity of the diagnostic tools.

\begin{tabular}{|c|c|c|c|c|c|}
\hline Diagnostic tools & Sensitivity (\%) & Specificity (\%) & PPV $(\%)$ & NPV (\%) & Accuracy $(\%)$ \\
\hline Morphology score & 89.0 & 100.0 & 100.0 & 97.0 & 97.0 \\
\hline Doppler score & 55.6 & 95.5 & 83.0 & 84.0 & 84.0 \\
\hline Doppler+morphology score & 100.0 & 95.5 & 90.0 & 100.0 & 96.6 \\
\hline
\end{tabular}

\section{DISCUSSION}

The present study showed that the use of color Doppler in addition to ultrasonography and ultrasound morphological study yields better characterization of ovarian masses and improve the diagnosis of malignant and benign tumors as well. Despite the numerous therapeutic protocols implemented in several studies; yet, ovarian cancer remains one of the most common cause of death among women. ${ }^{3}$ This is attributed to two unfavorable events. On one hand, ovarian cancer does not show symptoms in the early stage; hence, the diagnosis in most cases is made late. On the other hand, the treatments implemented are less effective in the advanced stages. ${ }^{2}$ Consequently, the only effective approach to this pathology remains the identification of the disease in the initial stages since survival rate would increase up to $70 \% .^{3}$

Both transabdominal and transvaginal pelvic ultrasonography are instrumental techniques widely used in ascertaining female neoplasms to identify the size and morphology of adnexal masses. ${ }^{9,14}$ They allow to evaluate some morphological parameters of the masses such as volume, wall thickness, their structure and possible presence of vegetation, possible presence of septa and their thickness, as well as echogenicity and content. ${ }^{7,11}$ However, it is evident that this method is not able, on the basis of the morphological characteristics alone, to distinguish between benign and malignant tumors, especially in early stages..$^{11}$ It has been reported that the sensitivity of morphologic analysis with ultrasound in predicting malignancy in pelvic tumors ranged between $87 \%$ and $100 \%$ whereas the specificity ranged between $45 \%$ and $95 \% .^{11,14-16}$ In this study, the sensitivity of this technique was $89.0 \%$ while the specificity was $100.0 \%$.

The addition of color Doppler imaging was found to improve the characterization of adnexal masses by means of vascularization through blood flow measurements obtained from tumor vessels. By this, the possibilities of making a correct diagnosis of malignancy or benignity are increased. ${ }^{11}$ The use of color Doppler to differentiate a benign from a malignant tumor is based on the observation of the remarkable the vascularization in the malignant masses with low resistance flows and high velocity. Resistance index $<0.4$ and pulsatility index $<1.0$ generally indicate malignancy. ${ }^{14}$

The analysis of our results showed that $8(19.0 \%)$ of the cases in group A were malignant compared to 7 (21.9\%) in group B. In group A, we did not have any false positive but we had one case of false negative result due to an ovarian borderline carcinoma that had a morphological score of 9 and which could account for the relatively low sensitivity reported by our study. Borderline tumors make up to $20 \%$ of epithelial ovarian tumors. ${ }^{17}$ These neoplasms are characterized by a relatively good prognosis compared to other ovarian tumors since, although showing various degrees of cytological atypia, they do not present any destructive stromal invasion even if they can be associated with extraovarian implants. ${ }^{17}$ Often bilateral, they mainly consist of mono or multilocular cysts, often with a thickened wall, and with endophytic or confluent papillary formations. Unlike carcinomas, they have no significant bleeding. ${ }^{17}$ Although the use of high frequency transvaginal probes provides information regarding the appearance and characteristics of pelvic masses and to evaluate the possibility of differentiating malignant forms from benign ones; however, there is a certain degree of overlap. The need has therefore arisen to seek further parameters capable of differentiating the benign from the malignant ones and in particular borderline tumors.

Therefore, one of the methods to detect these differences is to evaluate vascularization and resistance of blood flow. ${ }^{17}$ Gangopadhyay et al reported a sensitivity of $100 \%$ and specificity of $57.9 \%$ for mixed masses. ${ }^{9}$ In the present study, the color Doppler alone (group B) had a sensitivity of $55.6 \%$ and a specificity of $95.5 \%$. The low sensitivity obtained could be explained by the presence of four false negative cases: two mucinous adenocarcinomas, in which there was no highlighted flow and were evaluated as malignant masses at the morphological scale, a metastatic tumor, and a fibroleiomyosarcoma in which a flow with $\mathrm{RI}=0.5$ was detected. After examining in details the causes that interfered with a correct detection of the flow by color Doppler we found that the two adenocarcinomas were larger than $10 \mathrm{~cm}$ and predominantly cystic in structure, the metastatic tumor was associated with abundant presence of ascites $(>1000 \mathrm{ml})$ and leiomyosarcoma was essentially solid. These factors contribute to an altered detection of the Doppler because the large tumor masses have, if predominantly solid, an extensive area of central necrosis in which it is not possible to detect the presence of flows. ${ }^{18}$ We also assessed the peak systolic velocity by finding low values in the benign masses $(6.4 \mathrm{~cm} / \mathrm{sec})$ and high values in the malignant swellings $(17.7 \mathrm{~cm} / \mathrm{sec})$. These data agree with those of Sehgal. ${ }^{11}$ The low specificity obtained in our study could be attributed to a false positive case with a morphological score of 10 and an RI of 0.37 detected in the solid part of a predominantly cystic formation revealed on histological examination as a mucinous adenofibroma. On the basis of these results, having found a certain disparity between the 
morphological parameters and flow parameters, we combined the morphological score and Doppler outcome obtaining a sensitivity of $100.0 \%$ and a specificity of $95.5 \%$. The flow rates observed in malignancies seem variable and probably depend on various factors including the rate of tumor growth and the presence or absence of vascular necrosis.

Moreover, it is known that the vascularization of the tumor is heterogeneous. This is not only related to the type of tumor, but also to its rate of growth and its localization. ${ }^{11,14}$ Thus, it is essential, in order to improve the diagnostic accuracy of ovarian masses, to combine the morphological score with a flow score. ${ }^{11,15}$

\section{CONCLUSION}

In our experience, differentiating between benign and malignant pathology on the basis of the morphological evaluation alone, even if standardized with a score, or of the Doppler evaluation alone is not always satisfactory. We believe that the ultrasound study of pelvic masses with the combination of color Doppler and morphological study improves the diagnosis of pelvic tumors. The color Doppler adds a further evaluation criterion in malignant pathology, while in benign pathology it increases the diagnostic reliability of the ultrasound. Above all, it could represent the best test available for early diagnosis of the disease.

\section{Funding: No funding sources}

Conflict of interest: None declared

Ethical approval: The study was approved by the Institutional Ethics Committee

\section{REFERENCES}

1. Global Cancer Observatory. Lyon: International Agency for Research on Cancer.

2. Cabasag CJ, Butler J, Arnold M, Rutherford M, Bardot A, Ferlay J, et al. Exploring variations in ovarian cancer survival by age and stage (ICBP SurvMark-2): A population-based study. Gynecol Oncol. 2020;157:234-4.

3. Chandra A, Pius C, Nabeel M, Nair M, Vishwanatha JK, Ahmad S, et al. Ovarian cancer: Current status and strategies for improving therapeutic outcomes. Cancer Med. 2019;8:7018-31.

4. Momenimovahed Z, Tiznobaik A, Taheri S, Salehiniya H. Ovarian cancer in the world: epidemiology and risk factors. Int J Women Health. 2019;11:287-99.

5. Abramowicz JS, Condous G, Timmerman D. Ovarian mass-differentiating benign from malignant. Why the International Ovarian Tumour Analysis rules should be implemented in Australasia. Australas J Ultrasound Med. 2018;21:121-4.

6. Henderson JT, Webber EM, Sawaya GF. Screening for ovarian cancer: updated evidence report and systematic review for the US Preventive Services Task Force. JAMA. 2018;319:595-606.

7. Jacobs IJ, Menon U. Progress and challenges in screening for early detection of ovarian cancer. Mol Cell Proteomics. 2004;3:355-66.

8. Niazi M. Transabdominal vs transvaginal sonography-comparison in pelvic pathologies. JRMC. 2015;19:223-6.

9. Gangopadhyay S, Chakraborty P, Singh A. How successful is color flow mapping and pulsed Doppler studies in predicting histologically confirmed benign and malignant adnexal masses in perimenopausal women. Int J Reprod Contracept Obstet Gynecol 2017;6:5440-7.

10. Blanco EC, Pastore AR, Fonseca AM, Carvalho FM, Carvalho JP, Pinotti JA. Color Doppler sonography with contrast in the differentiation of ovarian tumors. Rev Hosp Clín Fac Med S Paulo. 2003;58:185-92.

11. Sehgal N. Efficacy of color doppler ultrasonography in differentiation of ovarian masses. J Midlife Health. 2019;10:22-8.

12. Tongsong $\mathrm{T}$, Wanapirak C, Neeyalavira V, Khunamornpong S, Sukpan K. E-flow doppler indices for prediction of benign and malignant ovarian tumors. Asian Pac J Cancer Prev. 2009;10:139-42.

13. Sassone AM, Timor-Tritsch IE, Artner A, Westhoff C, Warren WB. Transvaginal sonographic characterization of ovarian disease: evaluation of a new scoring system to predict ovarian malignancy. Obstet Gynecol 1991;78:70-6.

14. Khurana I, Satia MN. Preoperative evaluation of ovarian masses with color Doppler and its correlation with pathological finding. Int $\mathrm{J}$ Reprod Contracept Obstet Gynecol. 2016;5:2084-92.

15. Shah D, Shah S, Parikh J, Bhatt CJ, Vaishnav K, Bala DV. Doppler ultrasound: a good and reliable predictor of ovarian malignancy. J Obstet Gynecol India. 2013;63:186-9.

16. Khalaf LM, Desoky HH, Seifeldein GS, Al-Sharkawy M, Sayed MM, Ahmed S, et al. The diagnostic efficacy of Gynecology Imaging Reporting and Data System (GI-RADS): single-center prospective crosssectional study. Egypt J Radiol Nucl Med. 2019;50:19.

17. Fischerova D, Zikan M, Dundr P, Cibula D. Diagnosis, treatment, and follow-up of borderline ovarian tumors. Oncologist. 2012;17:1515-33.

18. Kurjak A, Kupesic S, Shalan H, Jukic S, Kosuta D, Ilijas M. Uterine sarcoma: a report of 10 cases studied by transvaginal color and pulsed Doppler sonography. Gynecol Oncol. 1995;59:342-6.

Cite this article as: Ghazal K, El Hasan J, Hijasi H, Koulaima E. Accuracy of ultrasonography and color Doppler in diagnosis of ovarian masses and its correlation with histopathological findings. Int $\mathbf{J}$ Reprod Contracept Obstet Gynecol 2020;9:4812-9. 